\title{
Nonlinear evolution of the parametric instability: numerical predictions versus observations in the heliosphere
}

\author{
F. Malara, L. Primavera, and P. Veltri \\ Dipartimento di Fisica, Università della Calabria, and Istituto Nazionale per la Fisica della Materia, Unità di Cosenza, \\ I-87030 Arcavacata di Rende, Italy.
}

Received: 26 July 2000 - Revised: 9 January 2001 - Accepted: 22 January 2001

\begin{abstract}
Low-frequency turbulence in the solar wind is characterized by a high degree of Alfvénicity close to the Sun. Cross-helicity, which is a measure of Alfvénic correlation, tends to decrease with increasing distance from the Sun at high latitudes as well as in slow-speed streams at low latitudes. In the latter case, large scale inhomogeneities (velocity shears, the heliospheric current sheet) are present, which are sources of decorrelation; yet at high latitudes, the wind is much more homogeneous, and a possible evolution mechanism is represented by the parametric instability. The parametric decay of an circularly polarized broadband Alfvén wave is then investigated, as a source of decorrelation. The time evolution is followed by numerically integrating the full set of nonlinear MHD equations, up to instability saturation. We find that, for $\beta \sim 1$, the final cross-helicity is $\sim 0.5$, corresponding to a partial depletion of the initial correlation. Compressive fluctuations at a moderate level are also present. Most of the spectrum is dominated by forward propagating Alfvénic fluctuations, while backscattered fluctuations dominate large scales. With increasing time, the spectra of Elsässer variables tend to approach each other. Some results concerning quantities measured in the high-latitude wind are reviewed, and a qualitative agreement with the results of the numerical model is found.
\end{abstract}

\section{Introduction}

In the ecliptic plane, which represents the region of the interplanetary medium most widely studied in space experiments, the solar wind is structured in high and low speed streams and is extremely variable. The heliospheric current sheet separating the global solar magnetic polarities is embedded within the low speed wind $(300-400 \mathrm{~km} / \mathrm{sec})$, while the high speed wind originates from open magnetic field lines regions, such as coronal holes. At high latitudes, recently studied by the Ulysses mission, the wind consists of a remarkably homoge-

Correspondence to: F. Malara (malara@fis.unical.it) neous (at least at solar minimum) flow with wind speed in the range $750-800 \mathrm{~km} / \mathrm{sec}$.

Large amplitude fluctuations are ubiquitously present, with frequency $f$ lower than the ion-cyclotron frequency, extending over a very wide range

$10^{-6} \mathrm{~Hz}<f<1 \mathrm{~Hz}$

in which fluctuations display a power law spectrum

$E(k) \propto k^{-\alpha}$

with spectral indices $\alpha$, comprised between 1 and 2 (Coleman, 1968; Belcher and Davis, 1971; Grappin et al., 1990; Marsch and Tu, 1990; Goldstein et al., 1995). This fact seems to be the signature of a fully developed MHD turbulence, resulting from a non-linear energy cascade (Dobrowolny et al., 1980).

Another relevant feature is that fluctuations are, in some sense organized (Veltri, 1980), primarily in fast-speed streams, as well as in the polar wind. In particular, velocity $\delta \boldsymbol{v}$ and magnetic field $\delta \boldsymbol{B}$ fluctuations appear to be highly correlated (Belcher and Davis, 1971)

$\delta \boldsymbol{v} \simeq \frac{\sigma \delta \boldsymbol{B}}{\sqrt{4 \pi \rho}} \quad$ with $\quad \sigma= \pm 1$

( $\rho$ being the mass density). The sign of the correlation ( $\sigma=$ \pm 1 ) corresponds to Alfvén waves propagating away from the Sun. Moreover, compressive fluctuations have a low level: $\delta \rho / \rho \ll 1$ and $\delta|\boldsymbol{B}| /|\boldsymbol{B}| \ll 1$. It is worth noting that these properties ( $\delta \boldsymbol{v}$ and $\delta \boldsymbol{B}$ fully correlated and vanishing compressions) characterize a large-amplitude Alfvén wave. The latter is an exact solution of the nonlinear MHD equations, which propagates without distortion at the Alfvén speed, provided that the background is homogeneous. In other words, nonlinear effects, which are responsible for the generation of a turbulent cascade, would be inhibited for such a solution. Thus, the presence of an extended spectrum seems to be in contradiction with the high degree of correlation.

This paradox can be explained as follows: the main source of low frequency fluctuations is inside the critical point, 
where both inward and outward propagating modes should be present. In this situation, a turbulent spectrum would easily form. Inward modes would be filtered out at the critical point, leaving the turbulence essentially "frozen" in a purely Alfvénic state. While the situation is somewhat more complicated (Velli, 1992), this explanation is widely accepted.

However, observations (Roberts et al., 1987, 1990; Bavassano and Bruno, 1989; Grappin et al., 1990) show that the solar wind turbulence continues to evolve beyond the critical point. The normalized cross-helicity

$\sigma_{c}=2 \frac{\langle\delta \boldsymbol{v} \cdot \delta \boldsymbol{b}\rangle}{\left\langle\delta v^{2}\right\rangle+\left\langle\delta b^{2}\right\rangle}$

(with $\boldsymbol{b}$ the magnetic field normalized to the Alfvén speed) which measures the degree of Alfvénicity, decreases with increasing distance from the Sun. This indicates a progressive "pollution" of the Alfvénic state.

At low latitudes, this phenomenon is relevant, in particular, in regions where strong inhomogeneities of the background medium are present: velocity shears separating fast and slow speed streams, with the current sheet at magnetic sector boundaries. Theoretical models have been developed to study the effects of such large-scale inhomogeneities on the turbulence evolution (Roberts et al., 1991, 1992; Grappin and Velli, 1996; Stribling et al., 1996; Malara et al., 1996, 1997, 1999)(see also Malara, 1999, for a review). These studies indicates that such an evolution leads to a strong depletion of $\sigma_{c}$, as well as a generation of compressive fluctuations, both magnetosonic and of entropy. The results of these models are in good agreement with measurements in the solar wind.

In regions where the background plasma is relatively homogeneous, such as in low-latitude fast streams and in the high-latitude wind, a decrease of Alfvénicity, with increasing distance, is observed (Bavassano et al., 2000a,b), though much slower than in slow speed streams. In such cases, it has been suggested that parametric instability could play an important role in the observed evolution (Tu and Marsch, 1995; Malara et al., 1996; Bavassano et al., 2000a; Malara et al., 2000). In fact, the parametric decay process of an circularly polarized Alfvén wave leads to the growth of a daughter wave with a correlation opposite to that of the mother wave (backscattered Alfvén wave), thus producing a decrease in the initial correlation.

Parametric instability has been studied in a variety of circumstances: small $\beta$ values (Sagdeev and Galeev, 1969), arbitrary $\beta$ s (Goldstein, 1978), nonlinear effects in the time evolution (Hoshino and Goldstein, 1989). Malara and Velli (1996) studied the instability when the initial "pump" wave has a nonmonochromatic spectrum. This is particularly relevant to the case of Alfvénic turbulence in solar wind, where the Alfvén waves have a widely extended power law spectrum.

The nonlinear development of parametric instability of a broadband Alfvén wave has been recently studied by Malara et al. (2000). In the present paper, we will focus on the final state, reached after the instability saturation: final cross- helicity, final level of compressive fluctuations and final spectra. These results will be compared with observations in the high-latitude solar wind.

\section{Numerical model}

We solve numerically the fully compressible, nonlinear, MHD equations in a one-dimensional configuration:

$$
\begin{aligned}
& \frac{\partial \rho}{\partial t}+\frac{\partial\left(\rho v_{x}\right)}{\partial x}=0 \\
& \frac{\partial \boldsymbol{v}}{\partial t}+v_{x} \frac{\partial \boldsymbol{v}}{\partial x}=-\frac{1}{\rho} \frac{\partial(\rho T)}{\partial x} \boldsymbol{e}_{x}+\frac{1}{\rho} \boldsymbol{j} \times \boldsymbol{b}+\frac{1}{\rho S_{v}} \frac{\partial^{2} \boldsymbol{v}}{\partial x^{2}} \\
& \frac{\partial \boldsymbol{b}}{\partial t}=\nabla \times(\boldsymbol{v} \times \boldsymbol{b})+\frac{1}{S_{\eta}} \frac{\partial^{2} \boldsymbol{b}}{\partial x^{2}} \\
& \frac{\partial T}{\partial t}+v_{x} \frac{\partial T}{\partial x}+\left(\gamma_{a}-1\right) T \frac{\partial v_{x}}{\partial x} \\
& \quad=\left(\gamma_{a}-1\right) \rho\left[\frac{1}{S_{\kappa}} \frac{\partial^{2} T}{\partial x^{2}}+\frac{1}{S_{v}}\left(\frac{\partial v_{i}}{\partial x} \frac{\partial v_{i}}{\partial x}\right)^{2}+\frac{1}{S_{\eta}} j^{2}\right]
\end{aligned}
$$

Here $\rho, v$ and $T$ are the plasma density, velocity and temperature, respectively, while $\boldsymbol{b}$ represents the magnetic field and $\boldsymbol{j}=\boldsymbol{\nabla} \times \boldsymbol{b}$ is the current density; $x$ and $t$ are the space and time variables, respectively. All the quantities are normalized to characteristic values. $S_{v}$ and $S_{\eta}$ represent the viscous and resistive Reynolds numbers, respectively, while $S_{k}$ is a dimensionless number related to the thermal conductivity. $\gamma_{a}=5 / 3$ is the adiabatic index.

The equations have been solved in the domain $x \in[0,2 \pi]$, by using a pseudospectral numerical code. Periodic conditions are imposed at the boundaries of the simulation interval. Further details about the numerical code can be found elsewhere (Malara et al., 1996). The unit time is the Alfvén time, namely the time it takes an Alfvén fluctuation to cross the simulation domain. The initial condition consists of a nonmonochromatic, large amplitude Alfvén wave, polarized in the $y z$ plane, propagating on a uniform background magnetic field $\boldsymbol{b}_{0}$. The total field is chosen to have uniform intensity everywhere:

$\boldsymbol{b}(x, 0)=b_{0} \boldsymbol{e}_{x}+b_{1}\left\{\cos [\phi(x)] \boldsymbol{e}_{y}+\sin [\phi(x)] \boldsymbol{e}_{z}\right\}$

Here $b_{1}=0.5$ is the ratio between the amplitude of the mother wave and the background magnetic field $b_{0}=1$. The function $\phi(x)$ represents the phase of the pump wave:

$\phi(x)=k_{0} x+a \sum_{\substack{-N \\ k \neq 0}}^{+N}|k|^{-\alpha} e^{i \delta_{k}} e^{i k x}$.

split into a monochromatic $\left(k_{0} x\right)$ and a nonmonochromatic part. The parameter $a$ is the relative weight of the latter to the former. Hence, setting $a=0$ corresponds to an initial monochromatic wave while, for larger $a$, the spectrum becomes increasingly non-monochromatic. There is no simple 
relation between the spectrum of the phase $\phi(x)$ and that of the magnetic field, the dependence in the Eq. (8) is nonlinear. However, we found that the spectrum of $\boldsymbol{b}(x, 0)$ roughly decreases with a power law, up to the wavenumber $N$, followed by a much faster decay. The coefficients $\delta_{k}$ in (9) are random numbers in the range $[0,2 \pi]$, while $\alpha=1$.

The initial wave is Alfvénic, therefore, the velocity field is given by:

$\boldsymbol{v}(x, 0)=\frac{c_{A 0}}{b_{0}} \delta \boldsymbol{b}(x, 0)$

where $c_{A 0}=1$ is the Alfvén speed. Hereinafter, the $\delta$ operator indicates the fluctuating part of any quantity $f: \delta f=$ $f-<f>_{x}$. The initial density $\rho(x, 0)=1$ and temperature $T(x, 0)=T_{0}$ are uniform.

Such an initial condition is an exact solution of the Eqs. (4)-(7) in the ideal case $\left(S_{v}=S_{\eta}=S_{k}=\infty\right)$. Thus, if no other perturbation were present in the system, the initial wave would propagate without distortion in the $\boldsymbol{e}_{x}$ direction. An initial noise of amplitude $10^{-6}$ was added to the density in order to trigger the instability.

\section{Numerical results}

Useful quantities to describe the simulation results are the Elsässer variables $z^{ \pm}$:

$z^{ \pm}=\boldsymbol{v} \pm \frac{\boldsymbol{b}}{\sqrt{\rho}}$.

In particular, we consider the pseudo-energies associated with the fluctuations of $z^{ \pm}$, which are defined by

$E^{ \pm}(t)=\frac{1}{2}\left\langle\left|\delta z^{ \pm}(x, t)\right|^{2}\right\rangle_{x}$

and the related normalized cross-helicity:

$\sigma(t)=\frac{E^{+}(t)-E^{-}(t)}{E^{+}(t)+E^{-}(t)}$

The average amplitude of density and magnetic field intensity fluctuations are respectively defined by

$r(t)=\left\langle\left(\frac{\delta \rho(x, t)}{\rho_{0}}\right)^{2}\right\rangle_{x}^{1 / 2}$

$m(t)=\left\langle\left(\frac{\delta b(x, t)}{b_{0}}\right)^{2}\right\rangle_{x}^{1 / 2}$

It is also useful to consider how the energy distributes at the different spatial scales during the time evolution. We calculated the Fourier power spectra of both the Elsässer variables

$e_{k}^{ \pm}(t)=\frac{1}{2} z_{k}^{ \pm}(t) \cdot z_{k}^{ \pm *}(t)$,

and the density fluctuations

$e_{k}^{\rho}(t)=\frac{1}{2} \rho_{k}(t) \cdot \rho_{k}^{*}(t)$, where "*" indicates complex conjugate, and the $k$-th Fourier coefficient $f_{k}(t)$ of a given quantity $f(x, t)$ is defined by

$f(x, t)=\sum_{k=-N_{p} / 2}^{N_{p} / 2} f_{k}(t) \exp (i k x)$.

At the initial time, $E^{-} / E^{+} \ll 1$ (corresponding to $\sigma \simeq$ 1) and $r \ll 1$. The development of parametric instability determines the growth of both $E^{-}(t)$ and $r(t)$. A detailed description of numerical results has been given in Malara et al. (2000). Here, we give a summary, and then we will focus on a possible application to solar wind turbulence.

The time evolution of the system can be roughly divided in two stages. During the first stage, the amplitude of unstable modes is small; thus, nonlinear mode couplings are negligible. In this situation, the linear theory predicts an exponential growth of perturbation, both for a monochromatic (Goldstein, 1978) and for a non-monochromatic (Malara and Velli, 1996) initial wave. An approximately exponential growth is present in the time evolution of both $E^{-}(t), r(t)$ and $m(t)$ (Fig. 1), and it allows one to give an estimation of the growth rate, $\gamma$, in the simulation results. It is found that $\gamma$ depends on the amplitude $a$ of the non-monochromatic part, in the phase (9) of the initial perturbation: a wider spectrum typically corresponds to a lower growth rate, which is maximum when $a=0$. Moreover, for a given value of $a, \gamma$ changes when changing the coefficients $\delta_{k}$, which contributes to the determination of the phase $\phi(x)$; this effect is more relevant when the spectrum is wider. However, the value of $\gamma$ remains of the same order of magnitude as for a monochromatic wave. These features reproduce the results of linear theory, found by Malara and Velli (1996).

In the second stage of the time evolution, nonlinear couplings are no longer negligible. Their effect is first to slow down the exponential growth of unstable modes, and finally to saturate the instability. The state of the system after saturation (level of pseudo-energies $E^{+}$and $E^{-}$, of compressive fluctuations $r(t)$ and $m(t)$, spectra) depends upon the value of the plasma $\beta$, defined by

$\beta=\frac{\gamma_{a} T_{0}}{c_{A 0}}$

while it is mainly independent of the spectrum of the initial Alfvén wave.

For small values of $\beta(\beta=0.1-0.5), E^{-}(t)$ grows until it becomes $\simeq E^{+}(t)$, thus, at saturation, the normalized crosshelicity is $\sigma \simeq 0$. This means that the Alfvénic correlation of the initial wave is completely destroyed by the instability. Compressive fluctuations grow to a moderate level: in the final state $r(t) \simeq 0.2$ and $m(t) \simeq 0.05$. The spectra $e_{k}^{+}$and $e_{k}^{-}$of the Elsässer variables are superposed, and they follow an approximate power law, in a relatively wide wavenumber range. These features indicates that, at small $\beta$, the state of the system after saturation is characterized by strong nonlinear interaction among Alfvénic (propagating in both directions) and compressive fluctuations. All features character- 

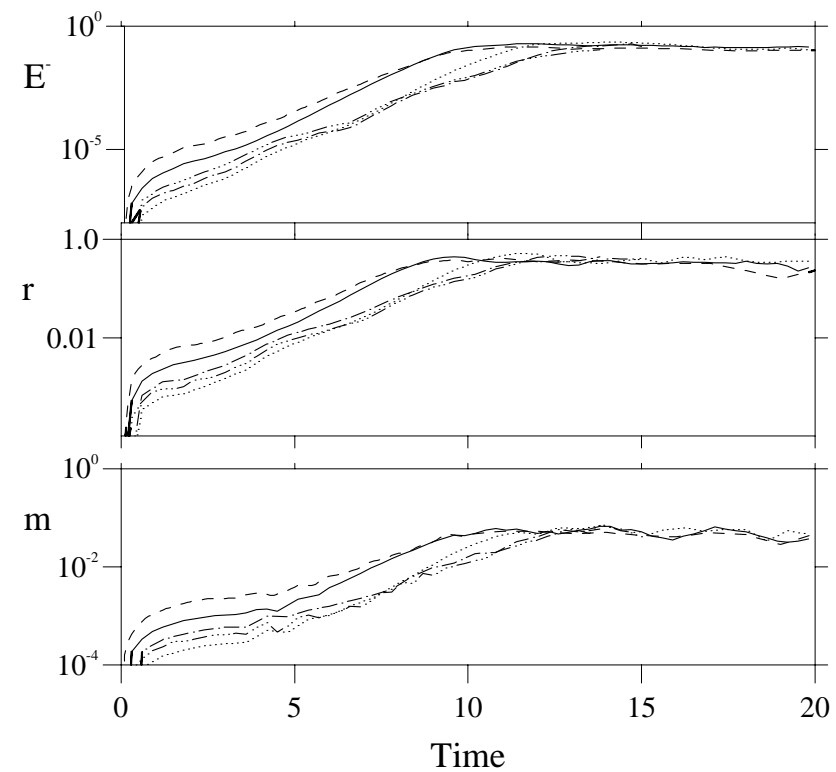

Fig. 1. Time evolution of $E^{-}(t)$ (upper panel), $r(t)$ (middle panel), and $m(t)$ (lower panel), in different configurations, with $0.2 \leq a \leq$ 0.8 and $\beta=0.1$.

izing both the initial state and the parametric process have disappeared.

Simulations of the parametric instability with intermediate values of $\beta$ ( $\beta \simeq 1)$ are more difficult to perform, because the growth rate of the instability decreases with increasing $\beta$. Nevertheless, values of $\beta \sim 1$ are more realistic to describe the solar wind plasma. For this reason, we performed some simulations at $\beta=1$.

In this case, the energy level $E^{-}$in the backscattered Alfvénic fluctuations, after the instability saturation, is of the same order as the energy $E^{+}$, but it remains lower than $E^{+}$ (Fig. 2, upper panel). Also, compressive fluctuations saturate at a level lower than that at $\beta=0.1$ : namely, $r(t) \simeq 0.03$ and $m(t) \simeq 0.01$ (Fig. 2, middle panel). The final value of the cross-helicity is $\sigma \simeq 0.5$ : it is lower than the initial value $(\simeq 1$ ), but it remains clearly positive (Fig. 2, lower panel). This indicates that, at $\beta \simeq 1$, parametric instability reduces the initial Alfvénic correlation, but it is unable to completely destroy it.

Parametric instability saturation is primarily determined by a decrease of the initial wave amplitude, which takes place when the Alfvénic daughter wave becomes large enough. The dispersion relation, which determines the wavelength of the interacting modes, depends on the amplitude of the Alfvén mother wave (Goldstein, 1978). When this amplitude changes, the interacting modes go off resonance, and the instability eventually stops. This effect becomes more relevant with increasing $\beta$; this is the reason why the final level of the cross-helicity $\sigma$ increases with increasing $\beta$. A more detailed discussion of this point is given in Malara et al. (2000).

Let us consider the time evolution of the spectra, during a typical simulation at $\beta=1$ (Fig. 3). The spectrum of
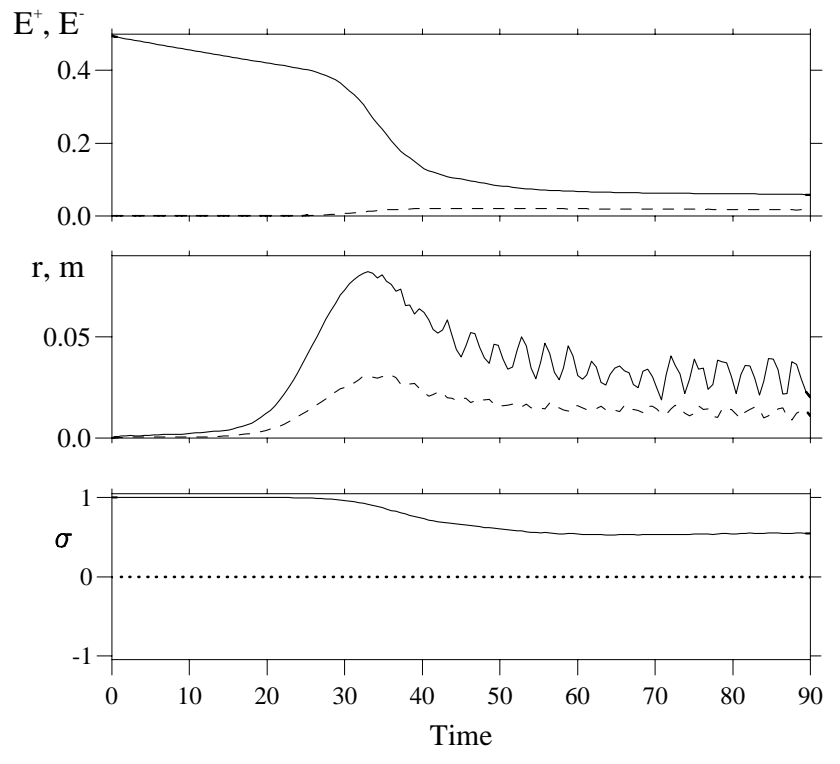

Fig. 2. A simulation at $\beta=1$ is represented. Upper panel: time evolution of $E^{+}(t)$ (full line) and of $E^{-}(t)$ (dashed line); middle panel: time evolution of $r(t)$ (full line) and of $m(t)$ (dashed line); lower panel: time evolution of $\sigma(t)$ (full line).

the mother wave $e_{k}^{+}$is peaked at $k_{0}=10$, but it extends in both ranges $k<k_{0}$ and $k>k_{0}$. Before the instability saturation $(t \leq 35)$, the spectra of backscattered Alfvénic fluctuations $e_{k}^{-}$and of density fluctuations $e_{k}^{\rho}$ are respectively peaked at $k_{A}^{-}=1$ and $k_{s}=11$ (even though lower peaks are present in the density spectrum at $k=1$ and in the $e_{k}^{-}$ spectrum at $k \simeq 11$ ). The wavenumbers of the main peaks are close to those excited by the parametric instability in the monochromatic limit (Goldstein, 1978; Malara et al., 2000). The spectra indicates that the mode interactions which characterize the parametric instability become strongly non-local in the $k$-space, with increasing $\beta$. The energy of the mother wave is transferred to backscattered modes at much larger wavelenghts $\left(k_{A}^{-} / k_{0} \sim 10\right)$, thus generating a sort of inverse cascade. Then, at variance with the small $\beta$ case, at saturation, the spectra $e_{k}^{+}$and $e_{k}^{-}$are completely different (Fig. 3, $t=35$ ): at small wavenumbers $e_{k}^{+} \sim e_{k}^{-}$, but in the range $k \gg 1$,it is still $e_{k}^{-} \ll e_{k}^{+}$. After saturation, with increasing time, the spectrum $e_{k}^{-}$gradually approaches $e_{k}^{+}$, though it remains lower than $e_{k}^{+}$. Moreover, the two spectra tend to become parallel for $k>k_{0}$ (Fig. $3, t=90$ ). At time $t=50$, a portion of the $e_{k}^{+}$spectrum has a slope close to $-5 / 3$ (Fig. 3 ) for $5 \leq k \leq 50$. At subsequent times, the spectrum becomes steeper, probably due to dissipation.

\section{Solar wind observations and comparison with the model}

It is interesting to try to compare the results derived from our numerical model with measures in the solar wind. Possible 


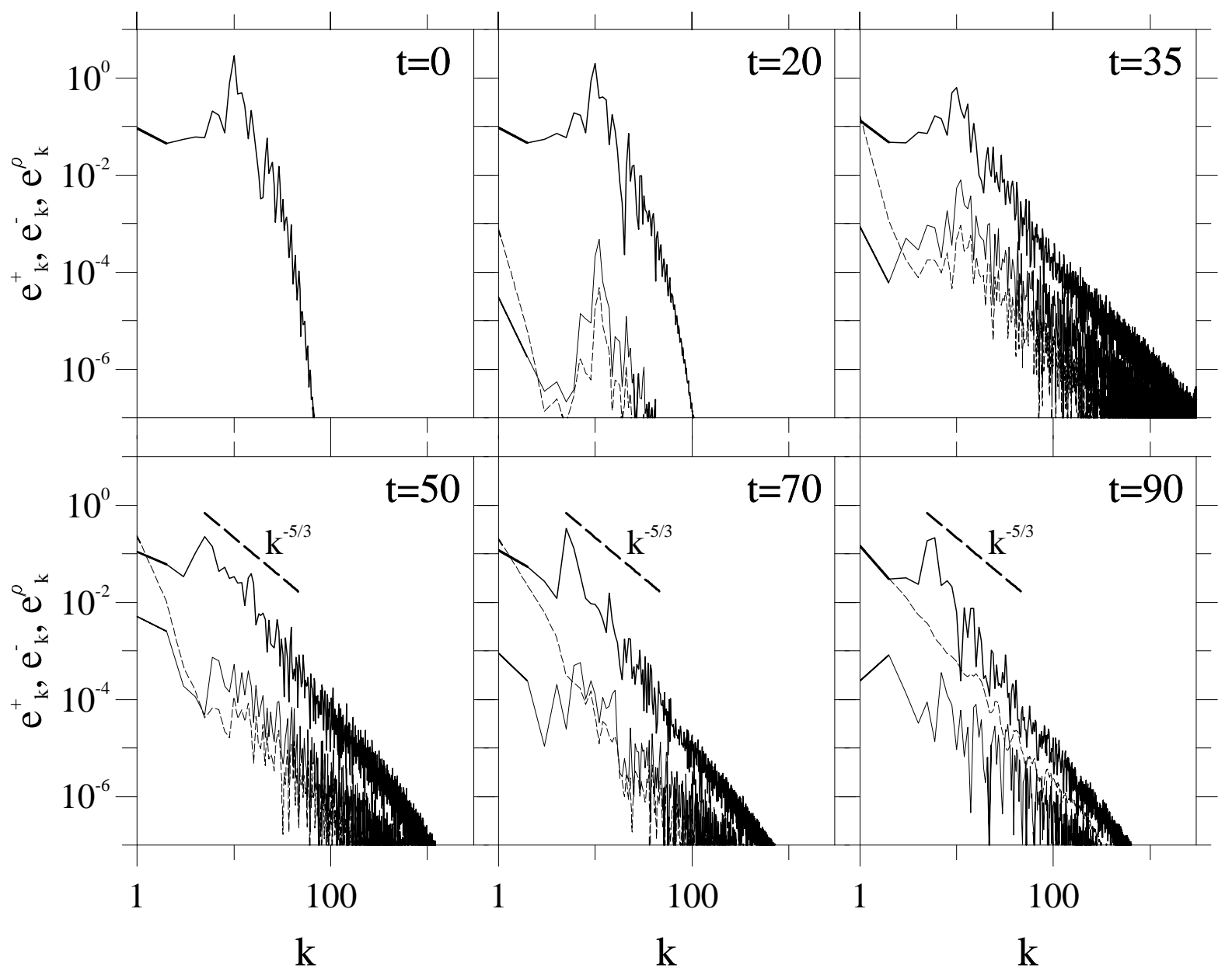

Fig. 3. Spectra $e_{k}^{+}$(thick line), $e_{k}^{-}$(dashed line) and $e_{k}^{\rho}$ (thin line) are represented at various times, for $\beta=1$. For $t \geq 50$ a power law spectrum $\propto k^{-5 / 3}$ is indicated for reference (thick dashed line).

effects of parametric instability in the evolution of the solar wind turbulence, such as the decrease of the cross-helicity and the growth of compressible fluctuations, compete with similar effects generated by the interaction of fluctuations with inhomogeneities of the medium at large scale (velocity gradients and/or the heliospheric current sheet). For this reason, the effects of a parametric decay process should be more easily detectable in regions where the solar wind is relatively homogeneous. This condition is verified at low latitudes only within fast-speed streams, and, with a better approximation, in the high-latitude wind.

Bavassano et al. (2000a,b) have recently calculated the dependence of the pseudo-energies of the Elsässer variable fluctuations on the distance $r$ from the Sun, in the solar wind. Their results are based on data from both Helios 2 and Ulysses spacecrafts. These data have been selected by choosing periods of steady and homogeneous wind, and fluctuations have been averaged on a time basis of 1 hour, i.e. within the Alfvénic frequency range. Thus, the results by Bavassano et al. $(2000 \mathrm{a}, \mathrm{b})$ can be used for a comparison with the results of our model.

In Fig. 4 , the pseudo-energy $e_{+}$and $e_{-}$in the above fre- quency range are represented: $e_{+}$and $e_{-}$are the energy of outward and inward propagating Alfvénic perturbations, respectively. Both $e_{+}$and $e_{-}$decrease with increasing distance $r$ from the Sun. Such a decrease is, in part, due to the solar wind expansion; this effect is not present in our model. However, the decrease of $e_{-}$is slower than that of $e_{+}$; correspondingly, the normalized cross-helicity $\sigma$, which is $\simeq 1$ close to the Sun $(r=0.3 \mathrm{AU})$, decreases with increasing $r$. However, this behaviour is found up to a distance of $r \simeq 2.5$; for larger distances, the energy of the outward propagating perturbations decay, with $r$ following a similar law as $e_{+}$(Bavassano et al., 2000a,b).

In Fig. 5 , the ratio $e_{-} / e_{+}$, calculated from the Ulysses data, is represented as a function of the distance $r$ (from Bavassano et al., 2000a). It is clearly seen that the ratio $e_{-} / e_{+}$increases with $r$, but it saturates, keeping a nearly constant value of $e_{-} / e_{+} \simeq 0.5$ for $r$ larger than $\sim 2-2.5 \mathrm{AU}$. This corresponds to a minimum value of the cross-helicity $\sigma_{\min } \simeq 1 / 3$.

The value of $\beta$ can be estimated using data from Ulysses measures: at the distance $r \simeq 2.5 \mathrm{AU}$ (where the ratio $e_{-} / e_{+}$ approximately saturates), the magnetic field intensity, the 


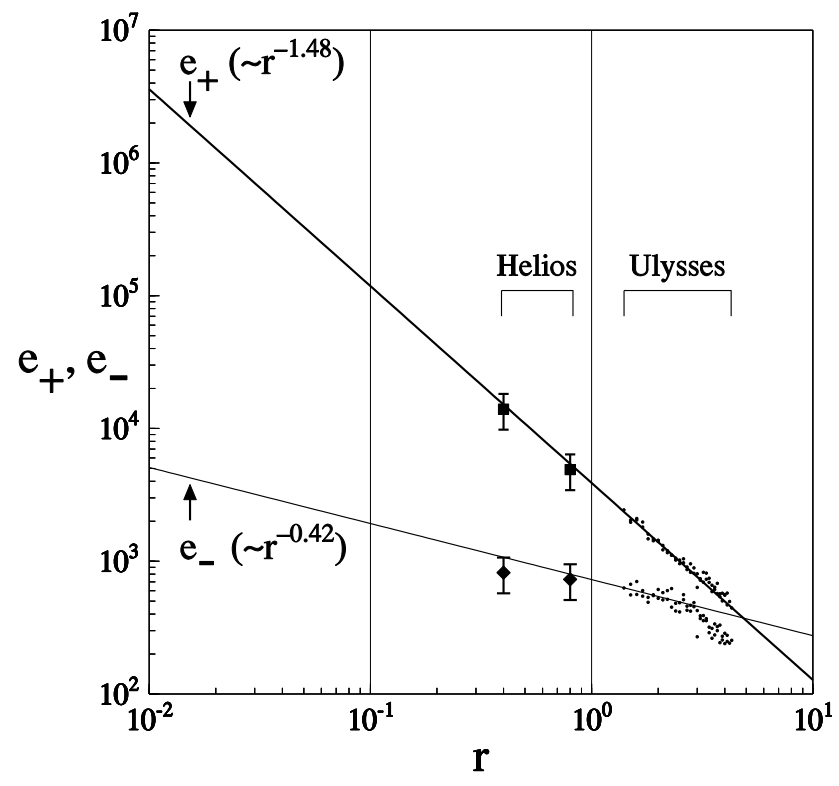

Fig. 4. The pseudo-energies $e_{+}$and $e_{-}\left(\right.$in $\left.\mathrm{km}^{2} \mathrm{~s}^{-2}\right)$ of outward and inward propagating Alfvénic fluctuations, averaged on a time basis of 1 hour, are represented as functions of the distance $r$ (in AU) from the Sun (from Bavassano et al., 2000a).

proton density and temperature are approximately given by $B \simeq 1.2 \mathrm{nT}, n_{p} \simeq 0.4 \mathrm{~cm}^{-3}$, and $T_{p} \simeq 1.5 \times 10^{5} \mathrm{~K}$, respectively. The corresponding estimation for $\beta$ is $\sim 1.2$, which is close to the value used in our simulation.

Goldstein et al. (1995) have calculated the spectra of the Elsässer variables in the high-latitude wind, using data from Ulysses spacecraft. At distance $r=2 \mathrm{AU}$, these authors find that the spectrum $e_{k}^{-}$is much lower than $e_{k}^{+}$in most of the considered frequency range $\left(10^{-6} \mathrm{~Hz} \leq f \leq 10^{-3} \mathrm{~Hz}\right)$, except at low frequencies, where $e_{k}^{-} \sim e_{k}^{+}$. For higher frequencies $\left(f \gtrsim 5 \times 10^{-5} \mathrm{~Hz}\right)$, the two spectra $e_{k}^{+}$and $e_{k}^{-}$are quasi-parallel (see Fig. 1b of Goldstein et al. (1995)). At larger distances from the Sun $(r=4 \mathrm{AU})$, the spectrum $e_{k}^{-}$ approaches $e_{k}^{+}$, and the two spectra are nearly parallel in a wider frequency range (Fig. 1c of Goldstein et al., 1995).

The relative growth of inward-propagating Alfvénic perturbations, with respect to outward propagating Alfvénic perturbations, within periods of homogeneous wind (far from sources of decorrelation due to inhomogeneities) could be due to a parametric decay process. The most striking feature in the observations is that the growth of the ratio $e_{-} / e_{+}$, calculated for hourly-averaged fluctuations, saturates at a maximum value $e_{-} / e_{+} \simeq 0.5$, indicating that the Alfvénicity of these perturbations is gradually reduced, but not completely destroyed. This behaviour is reminiscent of that found in our model, at intermediate values of $\beta$. The saturation level of cross-helicity in the numerical model is $\sigma_{\text {sat }} \simeq 0.5$ at $\beta=1$; the value found in the solar wind fluctuations is $\sigma_{\text {sat }} \sim 0.3-0.4$ (Bavassano et al., 2000a,b). Values of $\beta$ of the order of 1 (actually larger) have been calculated for the considered Ulysses data set. Similarities between the re-

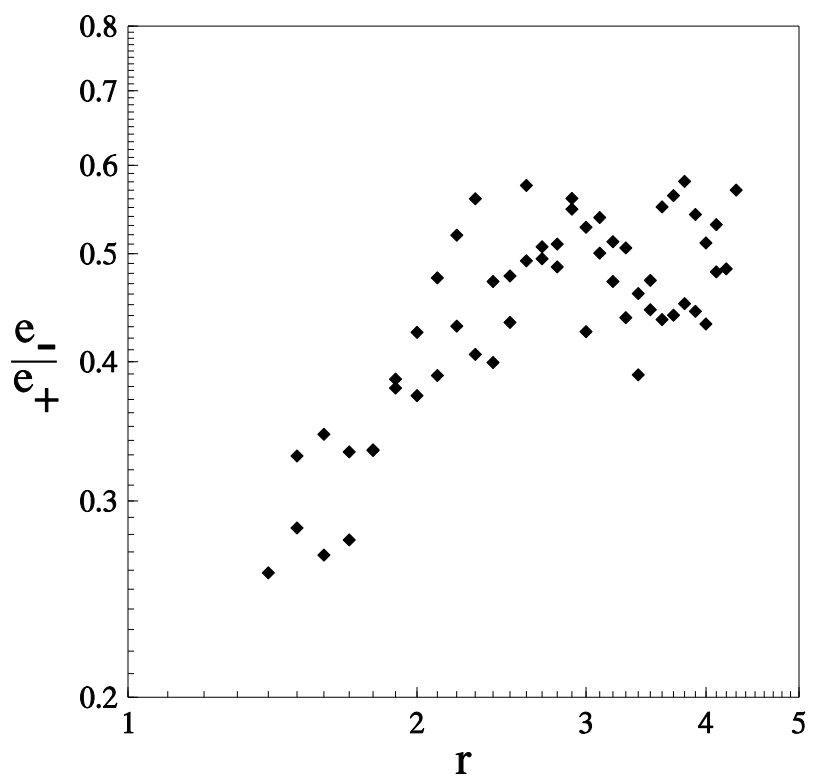

Fig. 5. The ratio $e_{-} / e_{+}$of pseudo-energies is represented vs. the distance $r$ (in AU) from the Sun (from Bavassano et al., 2000a).

sults of the numerical model and Ulysses measures can also be found in the evolution of the spectra of the Elsässer variables. In both cases: (i) before saturation $(t=35$ in the simulation and $r=2 \mathrm{AU}$ in the data) above a certain frequency the spectrum, $e_{k}^{-}$is parallel to $e_{k}^{+}$, with $e_{k}^{-} \ll e_{k}^{+}$ , while $e_{k}^{-} \sim e_{k}^{+}$at lower frequencies; (ii) with increasing time, or distance $r$ from the Sun, the two spectra approach each other.

\section{Discussion and conclusions}

The evolution of the low-frequency turbulence in the solar wind is characterized by a progressive decrease of Alfvénicity, with an increasing importance of both inward-propagating Alfvénic and compressive fluctuations. In the low-latitude wind, this phenomenon can be explained in terms of interactions between fluctuations and large-scale inhomogeneities, such as velocity shears and current sheets. Several studies, primarily based on numerical simulations, have been published (see, e.g. Malara, 1999; Veltri et al., 1999, for a review), which are able to explain different observed features in terms of the above mechanism.

At high latitudes, the wind is much more homogeneous. Nevertheless, a decrease of Alfvénicity is observed as well, though slower than at lower latitudes. A possible mechanism for such phenomenon could be the parametric instability, as suggested by Bavassano et al. (2000a) and, for high-speed stream turbulence, by Tu and Marsch (1995) and by Malara et al. $(1996,2000)$. In the present paper, we discussed the results of a numerical model describing the nonlinear evolution of parametric instability (Malara et al., 2000) with solar wind data. 
The model considers the evolution of an initial circularly polarized Alfvén wave with a broadband spectrum, perturbed by a low amplitude noise. The results show that the wave is always unstable; both oppositely correlated (backscattered) Alfvénic fluctuations and compressive waves grow, until the initial state is substantially modified. For $\beta \sim 1$ (which is a realistic value for the considered set of solar wind measures), the normalized cross-helicity $\sigma$, which is initially $=1$, after saturation, decreases to $\sigma \simeq 0.5$. This indicates that parametric instability reduces the initial Alfvénicity, but it is unable to completely destroy it. The spectra show that the energy of backscattered Alfvénic fluctuations is primarily concentrated at large scales (inverse cascade); this is characteristic of mode coupling at intermediate values of $\beta$ (Malara et al., 2000).

A comparison between the above results and data in the high-latitude solar wind has revealed some similarities, both in the behavior of the ratio $e^{-} / e^{+}$, which grows until it reaches a saturation value, and in the evolution of the spectra of the Elsässer variables. However, it is clear that the numerical model is a simplified representation of the turbulent Alfvénic state, measured in the high-latitude solar wind, and several features are not included in the model. First, the spectrum $e_{k}^{+}$of high-latitude solar wind fluctuations follows a power law, with no particular peaks (Goldstein et al., 1995). On the contrary, the spectrum $e_{k}^{+}$of the initial wave considered in the simulation, though it is not monochromatic, is dominated by a well-defined wavenumber $\left(k_{A}=10\right.$ at $\beta=1$ ). Even if no in situ measures are available at distances $r<0.3 \mathrm{AU}$, it is unlikely that the fluctuation spectrum very close to the Sun is dominated by a given frequency. Moreover, the spectra obtained in the simulations are steeper than those observed in the solar wind. This is, at least in part, due to dissipation, which is necessarily included in the numerical model. However, the simulation clearly demonstrates that an Alfvénic wave packet, containing a broadband of frequencies is parametrically unstable and the time evolution leads to a substantial reduction of the initial cross-helicity. Moreover, the inverse cascade associated with the parametric process increases the energy at wavenumbers smaller than $k_{A}$, so that the final spectrum has a shape much closer to a power law than the initial one, with a relevant reduction of the initial peak.

Other important limitations of the present model are the one-dimensionality and the simplified geometry, which does not allow one to describe the effects of the solar wind expansion. Work is in progress, both to study the evolution in 2D or 3D configurations, and to include solar wind expansion. Finally, arc-polarized wave packets are often measured in the solar wind. This kind of perturbation can be the result of nonlinear evolution of a linearly polarized Alfvén wave (Barnes and Hollweg, 1974). In this case, the variation of phase across the perturbation is typically $\Delta \phi<\pi$, thus it cannot be represented by the Eq. (9). We plan to include arc-polarized Alfvén waves in a future model.

In conclusion, due to the above limitations of the model, the comparison with solar wind data presented here must be regarded as being essentially qualitative, so the role played by parametric instability in the evolution of the Alfvénic turbulence of the solar wind is not yet fully clarified (see also a discussion by Tu and Marsch, 1995). Nevertheless, the results of our model, in which the constraint of monochromaticity has been relaxed, add new information on this point, suggesting that parametric instability could be responsible for the cross-helicity decrease, observed in regions where the wind is homogeneous and steady, as in the high-latitude wind.

Acknowledgements. The authors thank M. Velli for many useful discussions and suggestions on the simulation of parametric instability. They are also grateful to B. Bavassano, P. Pietropaolo and R. Bruno for clarifying discussions about the comparison with solar wind data. This work is supported by the Consiglio Nazionale delle Ricerche (CNR), contracts no. 98.00129.CT02, and no. 98.00148.CT02, the Ministero dell' Università e della Ricerca Scientifica e Tecnologica (MURST), the Agenzia Spaziale Italiana (ASI), contract no. ARS98-82, and the INTAS Open 97-1612 grant

\section{References}

Barnes, A. and Hollweg, J. V., Large-amplitude hydromagnetic waves, J. Geophys. Res., 79, 2302, 1974.

Bavassano, B. and Bruno, R., Evidence of local generation of Alfvénic turbulence in the solar wind, J. Geophys. Res., 94, 11977-11982, 1989.

Bavassano, B., Pietropaolo, E., and Bruno, R., On the radial decrease of Alfvénic fluctuations in the polar wind, in Magnetic fields and solar processes, proceedings of the 9th European Meeting on Solar Physics, A. Wilson Ed., ESA Publication Division, Noordwijk, The Netherlands, 1135-1139, 2000a.

Bavassano, B., Pietropaolo, E., and Bruno, R., On the evolution of outward and inward Alfvénic fluctuations in the polar wind, J. Geophys. Res., 105, 15959-15964, 2000b.

Belcher, J. W. and Davis, L., Large-amplitude Alfvén waves in the interplanetary medium, J. Geophys. Res., 76, 3534-3563, 1971.

Coleman, P. J., Turbulence, viscosity and dissipation in the solar wind plasma, Astrophys. J., 153, 371, 1978.

Dobrowolny, M., Mangeney, A., and Veltri, P., Fully developed anisotropic hydromagnetic turbulence in interplanetary space, Phys. Rev. Lett., 45, 144-147, 1980.

Goldstein, B. E., Smith, E. J., Balogh, A., Horbury, T. S., Goldstein, M. L., and Roberts, D. A., Properties of magnetohydrodynamic turbulence in the solar wind as observed by Ulysses at high heliographic latitudes, Geophys. Res. Lett., 22, 3393-3396, 1995.

Goldstein, M. L., An instability of finite amplitude circularly polarized Alfvén waves, Astrophys. J., 219, 700-704, 1978.

Grappin, R., Mangeney, A., and Marsch, E., On the origin of solar wind turbulence: Helios data revisited, J. Geophys. Res., 95, 8197-8210, 1990.

Grappin, R. and Velli, M., Waves and streams in the expanding solar wind, J. Geophys. Res., 101, 425-444, 1996.

Hoshino, M. and Goldstein, M. L., Time evolution from linear to nonlinear stages in magnetohydrodynamic parametric instabilities, Phys. Fluids B, 1, 1405-1415, 1989.

Malara, F., Theoretical aspects of MHD turbulence in the presence of large scale structures, in Solar Wind Nine, edited by S. R. Habbal, R. Esser, J. V. Hollweg, and P. A. Isenberg, AIP, 155$160,1999$. 
Malara, F., Primavera, L., and Veltri, P., Compressive fluctuations generated by time evolution of Alfvénic perturbations in the solar wind current sheet, J. Geophys. Res., 101, 21597-21617, 1996.

Malara, F., Primavera, L., and Veltri, P., Nature of the densitymagnetic-field-intensity correlation in the solar wind, Phys. Rev. E, 56, 3508-3514, 1997.

Malara, F., Primavera L., and Veltri, P., Effects of entropy inhomogeneity on density-temperature correlation in the solar wind, Phys. Rev. E, 59, 6023-6031, 1999.

Malara, F., Primavera, L., and Veltri, P. Nonlinear evolution of parametric instability of a large-amplitude nonmonochromatic Alfvén wave, Phys. Plasmas, 7, 2866-2877, 2000.

Malara, F. and Velli, M., Parametric instability of a large-amplitude nonmonochromatic Alfvén wave, Phys. Plasmas, 3, 4427-4433, 1996.

Marsch, E. and Tu, C.-Y., Spectral and spatial evolution of compressible turbulence in the inner solar wind, J. Geophys. Res., 95, 11945-11956, 1990

Roberts, D. A., Ghosh, S., Goldstein, M. L., and Matthaeus, W. H., Magnetohydrodynamic simulation of the radial evolution and stream structure of solar-wind turbulence, Phys. Rev. Lett., 67, 3741-3744, 1991.

Roberts, D. A., Goldstein, M. L., and Klein, L. W., The amplitudes of interplanetary fluctuations: stream structure, heliocentric distance, and frequency dependence, J. Geophys. Res., 95, 42034216,1990
Roberts, D. A., Goldstein, M. L., Klein, L. W., and Matthaeus, W. H., Origin and evolution of fluctuations in the solar wind: Helios observations and Helios-Voyager comparisons, J. Geophys. Res., 92, 12023-12035, 1987.

Roberts, D. A., Goldstein, M. L., Matthaeus, W. H., and Ghosh, S., Velocity shear generation of solar wind turbulence, J. Geophys. Res., 97, 17115-17130, 1992.

Sagdeev, R. Z. and Galeev, A. A., Nonlinear Plasma Theory Benjamin, New York, 1969.

Stribling, T., Roberts, D. A., and Goldstein, M. L., The evolution of Alfvénic perturbations in a three-dimensional MHD model of the inner heliospheric current sheet region, J. Geophys. Res., 101, 27603-27623, 1996.

Tu, C.-Y. and Marsch, E., MHD structures, waves and turbulence in the solar wind: Observations and theories, Space Sci. Rev., 73, $1-210,1995$.

Velli, M., On the propagation of ideal, linear Alfvén waves in radially stratified stellar atmospheres and winds, Astron. Astrophys., 270, 304-314, 1992.

Veltri, P., An observational picture of Solar Wind MHD Turbulence, Il Nuovo Cimento, C3, 45-55, 1980.

Veltri, P., Malara, F., and Primavera, L., Nonlinear Alfvén wave interaction with large-scale heliospheric current sheet, in Nonlinear MHD Waves and Turbulence, T. Passot, P.-L. Sulem, Eds., Lecture Notes in Physics, Springer, vol. 536, pp. 222-250, 1999. 